\title{
The Detection Method of Load Transient Characteristics Based on Second-order Statistics
}

\author{
Bing Qi , Hui Bai, Xin Wu \\ North China Electric Power University \\ School of Electric and Electronic Engineering \\ Beijing , China \\ Email:1020280347@qq.com
}

\begin{abstract}
In non-intrusive load monitoring, types and operation situation of a single load in cluster are obtained by analyzing the power signal at the electricity entrance. With the increasing complexity of large grid structure and the continuous development of smart grids, non-intrusive load monitoring is getting more important. In this paper, a load transient characteristics detection method based on second-order statistics is studied, the non-intrusive load monitoring system architecture involved in this paper is elaborated in detail. On the basis of the measured data load, load transient features are analyzed in-depth, simulation results are given.
\end{abstract}

Key words-non-intrusive; load transient characteristics; second-order statistics; autocorrelation function; auto-covariance function

\section{INTRODUCTION}

With the development of the smart grid, residential electricity load monitoring is particularly important. It is not only conducive to improve the load composition, reduce electricity cost, but also conducive to optimize the national electricity resources allocation.Literature [1] indicates that in traditional load monitoring, sensors and other hardware devices need to be installed in each monitored load point to get the different loads of energy data.A lot of time and money are spent on installation and maintenance, but still, this method cannotmeet the needs of the power system or spread among users.The concept of "Non-intrusive LoadMonitoring" (NILM) was first proposed by $\mathrm{Hart}^{[2]}$. In this monitoring system, monitoring equipment are required to be installed at the entrance of electricity,by monitoring and analyzing the voltage, current and other signal, types and operation situation of single load in load cluster can be obtained.Studies have shown that non-intrusive load monitoring system is an important development direction of future load monitoring, and with the continuous development of power systems, will derive a greater role and function [3]. Compared with the conventional load measurement method, the advantages of non-intrusive load monitoring systems are summarized below:

1) Easy to maintain:only need to install the meter at the power entrance;

2)Easy to collect data:every single piece of load information within the system can be easily collected without entering the system;

3)Easy to analysis data: data can be obtained and analyzed in the main station.

As the rapid development of measurement, communications, computer science and other technologies, the non-intrusive load monitoring systems analysis approach has undergone great changes.Analysis of transient characteristics of power load gradually become the focus of researchers, it is closely related to the physical tasks it performs.Usually, unique characteristics transient information will be presented during the process of switching different types of load.According to the transient character information, the internal load cluster decomposition and analysis can be achieved ${ }^{[4]}$.Furthermore, useful information can be obtained.To a certain extent, by applying the power load transient characteristics analysis, the limitation of load identification using steady-state load characteristic information can beovercome, load recognition rate can be improved.

In this paper, under the non-intrusive load monitoring environment, residential load electricity data are obtained, different characteristics of household loads are analyzed by using the load transient characteristic detection method based on second-order statistics, the foundation for further identification of research is laid.

\section{NON-INTRUSIVE LOAD MONITORINGSYSTEM}

The non-intrusive load monitoring system architecture in this study is shown in Figure 1. 


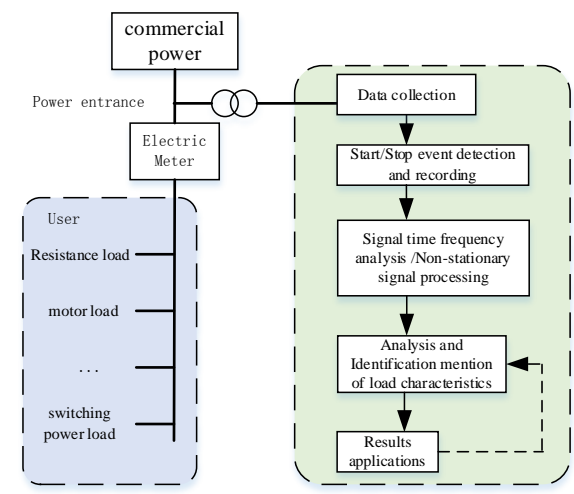

Fig.1 The non-intrusive load monitoring system architecture

1) Data collection: collect real-time voltage and current information at the power entrance side.

2) Start/Stop event detection and recording: By pre-process current/voltage signals, perform load start/stop event identification, recording start/stop events.

3)Signal time frequency analysis /Non-stationary signal processing:Select characteristic functionto expand signal. Combined with a large number of experimental data, separate the steady-state separation characteristics and transient characteristics of the signal, using 1-order, second-order and higher-order statistics method to process non-stationary signal.

4) Analysis and Identification mention of load characteristics: According to the result of "signaltime frequency analysis /non-stationary signal process", extract steady/transient characteristics of the load to analysis.Using mathematical analysis methods and efficient algorithms,it can make the machine automatically identify the load characteristics, identify the type of load and the operation it performed.

5) Results applications: can achieve load real-time energy consumption monitoring, abnormal alarm functions and data backhaul.

Different load power change and other information caused by the changing switch status are used to classify in non-intrusive load monitoring system. And the information is also used to identify or estimate each load type, operating conditions and related parameters and other information.Currently, real-time automatical remote residential power consumption information collection, intelligent two-way interactive services and other functions can be achieved.

Resistance load (load 1), motor load (load 2), switching power load (load 3, load 4) are being selected in the simulation experiment.The feasibility of load transient characteristics analysisusing second-order cumulant (autocorrelation function, auto--covariance function) is verified.

\section{TRANSIENT CHARACTERISTIC ANALYZING METHOD}

Non-intrusive load monitoring and analysis methods can be divided into two categories ${ }^{[5]}$, the analysis method based on load steady-state characteristics and the one based on load transient characteristics.Among them, the steady-state characteristics mainly refer to the load steady-state fundamental wave, harmonic power and other features.The main characteristics of transient load mainly refer to variation rule of open momentary voltage, current and etc., such as transient waveform and its structure.

In the early 1990s, the load identification method based on the load switching transient process information was proposed by Steven Leeb and his team of scholars from the Massachusetts Institute of Technology ${ }^{[6]}$.According to the research, load transient current signal can be used to identify various types of loads.Steven Leeb found that transient mode has unique features when the load is switched on [7], so simulated spectral envelope analyzer is designed to estimate the load active power, reactive power and harmonic power and other information.According to the principle above, the corresponding transient detection andload classification algorithms are determined. Digital spectrum envelope analyzer are developed by Shaw [8], and the improved Discrete Fourier Transform (DFT) algorithm [9] based on waveform sample for NILM system are presented, in order to achieve real-time load monitoring.The envelope used to extract load switching transient process load currentbased on Kalman filter algorithm is proposed in literature [7].Information spectrum analyzing method based on electrical equipment transientswitching is proposed in literature $[10,11]$, to achieve load component decomposition. Load component decomposition to voltage disturbance based on electrical equipment transientswitching is proposed in literature [12], its limitation is that when the power load number is big or the device is switching frequently, the accuracy of the method can't be guaranteed.

During the process of load feature analysis, the problem of insufficient informationwill be encountered, such as: an element with the same steady-state value may have different transient starting current.In response to these and other issues, as a complement to the steady-state characteristics, information is provided by the transient characteristics for further study. For example: transient value will not exist or only in a short time (less than $50 \mathrm{~Hz}$ period) in resistive load during switching process.Long-term transient value can be generated in the pumpor other equipment drove by electric motor; other motor-driven appliances such as fans, washing machines, blenders and other equipment, the start-up transient value is very small.Electronic appliances (TVs, VCRs, Computers) is characterized by a short 
but large amplitude transient start value; fluorescent lamp has a long second-order transient values, etc.In summary, the analysis of transient load characteristics is essential for NILM system.

\section{The Detection Method of LoAd Transient CHARACTERISTICS BASED ON SECOND-ORDER STATISTICS}

The transient characteristics of residential load for detection, second-order statistics method is used in this paper, the autocorrelation function and covariancefunction in time domain. The detection method ofload characteristicsbased on second-order statistics, for the advantages of fast operation speed, simple implementation, and reliable running, the analysis of the transient characteristics of the residents load is a representative method.

\section{A. The Autocorrelation Function in Time Domain}

It is assumed that second-order joint probability density function $p\left(x_{1}, x_{2} ; t_{1}, t_{2}\right)$ of nonstationary random signal $X_{1} 、 X_{2}$, its autocorrelation function is defined as

$$
\begin{aligned}
& r_{x X}\left(t_{1}, t_{2}\right)=E\left[X\left(t_{1}\right)_{X}\left(t_{2}\right)\right]= \\
& \int_{-\infty}^{\infty} \int_{-\infty}^{\infty} x_{1} x_{2} p\left(x_{1}, x_{2} ; t_{1}, t_{2}\right) \mathrm{d} x_{1} d x_{2}
\end{aligned}
$$

According to the original definition, the autocorrelation function has the symmetry: $r_{x X}\left(t_{1}, t_{2}\right)=r_{X X}\left(t_{2}, t_{1}\right)$ 。

Similarly, $r_{x x}\left(t_{1}, t_{2}\right)$ can be estimated by $N$ samples $X_{i}(t)(i=1,2, \ldots, N)$, that is

$$
\hat{\mathrm{r}}_{x X}\left(t_{1}, t_{2}\right)=\frac{1}{N} \sum_{\mathrm{i}=1}^{N} X_{i}\left(t_{1}\right)_{X_{i}}\left(t_{2}\right)
$$

If $t_{1}$ is fixed, changing $t_{2}=t_{1}+\tau, \tau$ is time Delay, then

$$
r_{x X}\left(t_{1}, t_{1}+\tau\right)=r_{x X}\left(t_{1}, \tau\right)=E\left[X\left(t_{1}\right) X\left(t_{1}+\tau\right)\right]
$$

It is a function of ${ }^{t}=t_{1}$, and is a function of the time delay $\tau$. But it is not even function of $\tau$, because of nonstationary random signal $r_{x x}\left(t_{1}, t_{1}+\tau\right) \neq r_{x x}\left(t_{1}, t_{1}-\tau\right)$. Thus, the Fourier Transform of $r_{x x}\left(t_{1}, t_{1}+\tau\right)$ is not a real function so that the physical interpretation of the spectrum makes it difficult. Therefore, the autocorrelation function of the nonstationary random signal is given another form below:

$$
r_{x X}(t, \quad \stackrel{\operatorname{def}}{=} E[X(t+\tau / 2) X(t-\tau / 2)]
$$

Clearly, this definition of the autocorrelation function with even features, that is

$$
r_{X X}(t, \tau)=r_{x X}(t,-\tau)
$$

And when $\mathrm{x}(t)$ is a real signal, $r_{x x}(t, \tau)$ is the real function, so that it can remain solid even Fourier transform characteristics desired. Thus, the autocorrelation function is called symmetric, can be applied to the analysis and processing of non-stationary random signals.

\section{B. The Covariance Function in Time Domain}

Similarly, since the covariance function is defined as

$$
\left.C_{\text {xx }}\left(t_{1}, t_{2}\right)=E\left\{X\left(t_{1}\right)-m_{X}\left(t_{1}\right)\right]\left[X\left(t_{2}\right)-m_{x}\left(t_{2}\right)\right]\right\}
$$

In particular, the relationship between the $C_{\mathrm{xx}}\left(t_{1}, t_{2}\right)$ and $r_{x x}\left(t_{1}, t_{2}\right)$ is

$$
C_{\mathrm{xx}}\left(t_{1}, t_{2}\right)=r_{x x}\left(t_{1}, t_{2}\right)-m_{x}\left(t_{1}\right) m_{x}\left(t_{2}\right)
$$

\section{Detection Procedure}

According to the measured data of a large number of residential loads, non-intrusive load monitoring as standards for load transient characteristic detection can be divided into two steps.The first step: using the detection means, collecting the load voltage, current, power, switching events and other information;The second step: using the calculation method of second-order cumulant (autocorrelation function, auto-covariance function) is used to data processing, drawingconclusions through analysis.

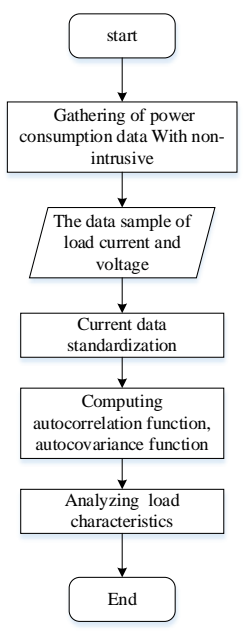

Fig.2 The flow chart of detection method of load transient characteristics.

\section{EXPERIMENTAL VERIFICATION}

\section{A. Experimental Environment}

The electricity data collection terminal of residents load involves data acquisition card, transformers, filter circuit and isolation circuit.

During the experiment, firstly, the hardware environment should be set (including all types of typical electricity load).And electricity data for single 
user load equipment should be collected at the entrance of load device and the entrance ofcommercial power. Secondly, thedata preprocessing is helpful to facilitate later analysis.Finally, import the processed measured data i nto analysis software, analysis the charactaristics usin g load characteristics detection algorithms.

Load 1, load 2, load 3are being selected in the simulation experiment. Load1and load2 is put into operation in the beginning of the extraction of 100 sampling points are calculated. Load3is about 3300 sampling points are calculated.

\section{B. Simulation Results}

The autocorrelation function , the auto-covariance function are used in this section,simulation results of load characteristics areobtained.

1) Current signal in time domain: In the following three figures, the current time-domain signal is given, including load 1 , load 2 and load 3.

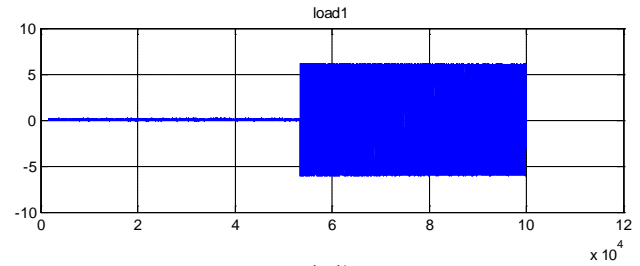

Fig.3 Current signal of load 1 in time domain.

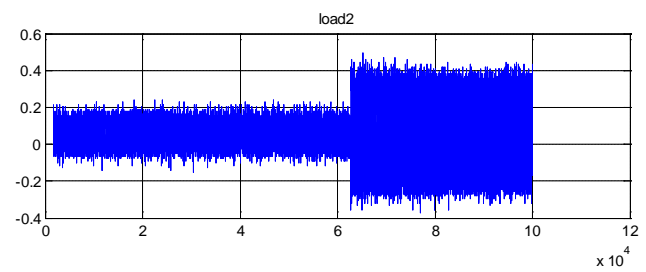

Fig.4 Current signal of load 2 in time domain.

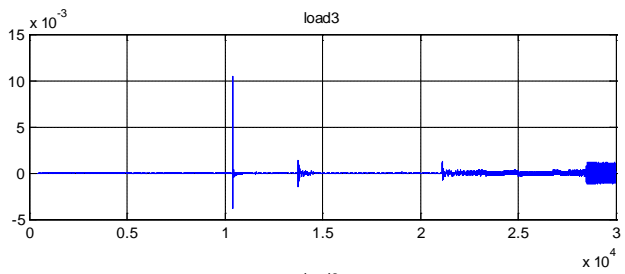

Fig.5 Current signal of load 3 in time domain.

2) Probability density function

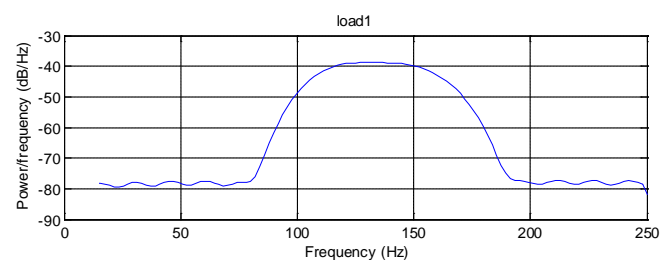

Fig.6Theprobability density function of load 1.

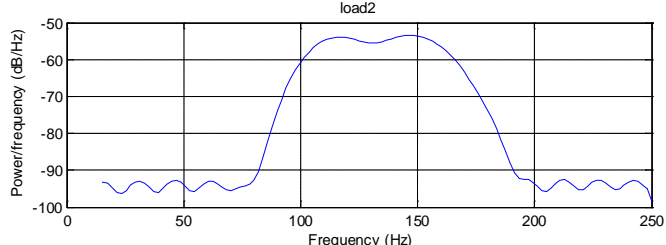

Fig.7Theprobability density function of load 2.

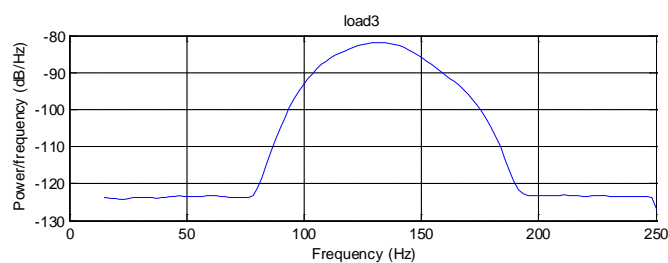

Fig.8Theprobability density function of load 3.

3) Autocorrelation function

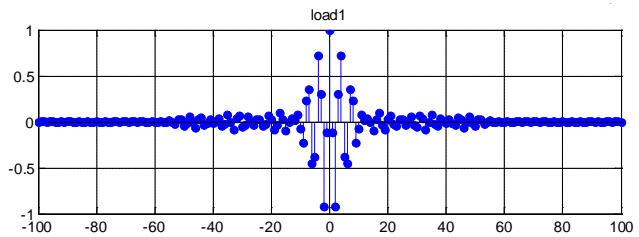

Fig.9 The autocorrelation function of load 1.

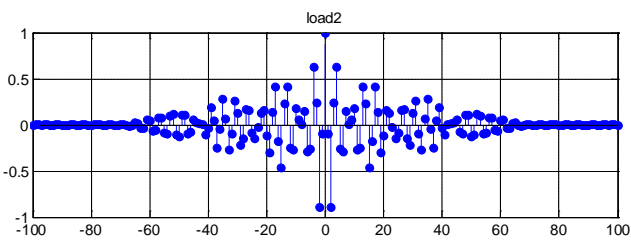

Fig.10 The autocorrelation function of load 2.

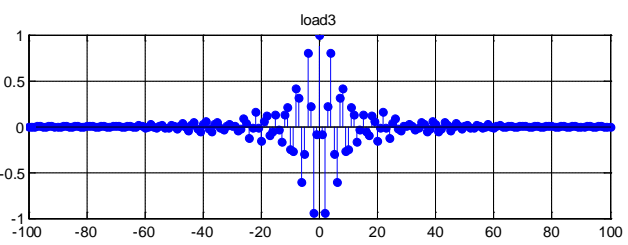

Fig.11 The autocorrelation function of load 3.

The autocorrelation function of current signal describes dependencebetween a moment and another moment, that is study time $t$ and $t+t^{\prime}$. Comparing these three figures, the autocorrelation function of there load are very different. Three kinds of load can easily be distinguished by using this method of statistics. 
4) Auto-covariance function

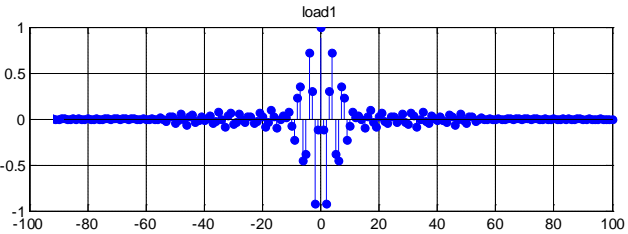

Fig.12 The auto-covariance functionof load 1.

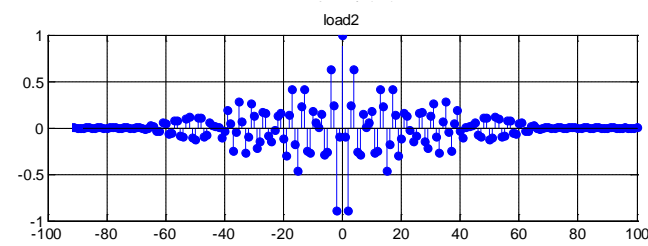

Fig.13 The auto-covariance functionof load 2.

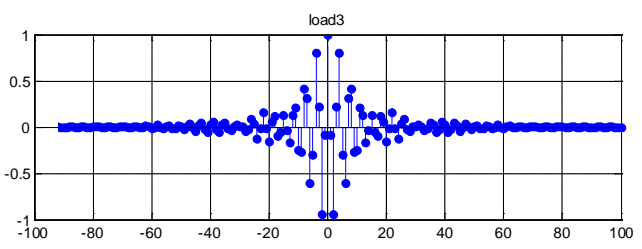

Fig.14 The auto-covariance functionof load 3.

Similarly, auto-covariancefunction of current signal describes the dependencebetween a moment and another moment, and three kinds of load can easily be distinguished.The auto-covariance function playsassisted role in terms of load identification.

\section{SUMMARY}

In this paper, under the non-intrusive load monitoring environment, residential load electricity data are obtained, different characteristics of residential loads are analyzed by using the load transient characteristic detection method based on second-order statistics, the foundation for further identification of research is laid. The test proved that the method for the different characteristics of the residential load has good effects. A theoretical basis of load feature library is provided in non-intrusive load monitoring. And how to classifysimilar loads of classification and appliance load optimal feature data extraction, further research is needed.

\section{REFERENCES}

[1] NIU Lulu. Non-intrusive Load Monitoring Based onTransient Characteristics[D].Tian Jin University, 2010.

[2] G.W.Hart, Nonintrusive Appliance Load Monitoring [J]. Proceedings of the IEEE,1992,80(12):1870-1891.

[3] Zoha A, Gluhak A, Imran M A, et al. Non -intrusive loadmonitoring approaches for disaggregated energy sensing: asurvey[J]. Sensors, 2012, 12(12): 16838-16866.

[4] Baranski, M.; Voss, J. Non -Intrusive Appliance LoadMonitoring Based on an Optical Sensor.In Proceedings of IEEEPower Tech Conference,Bolgona, Italy, 23-26 June 2003:8-16.
[5] Najmeddine H., El Khamlichi Drissi K., Pasquier C., Faure C., Kerroum K.,Diop A., Jouannet T., Michou M. State of art on load monitoring methods [C].In: IEEE 2nd International Power and Energy Conference, 2008: 1256-1258.

[6] Leeb S. B. A Conjoint Pattern Recognition Approach to Nonintrusive LoadMonitoring [Dissertation]. Cambridge: Massachusetts Institute of Technology,1993.

[7] Shaw S. R., Abler C. B., Lepard R. F., Luo D., Leeb S. B., Norford L.K.Instrumentation for High Performance Nonintrusive Electrical LoadMonitoring[J]. ASME Journal of Solar Energy Engineering, 1998,120(3):224-229.

[8] Shaw S. R. System Identification Techniques and Modeling for NonintrusiveLoad Diagnostics [D]. Cambridge: Massachusetts Institute of Technology,2000.

[9] Shaw S. R., Laughman C. R.A Kalman-Filter Spectral EnvelopePreprocessor[J]. Instrumentation and Measurement, IEEE Transactions on,2007, 56(5):2010-2017.

[10] Khan U.A., Leeb S.B., Lee M.C. A Multiprocessor for Transient EventDetection[J]. Transactions on Power Delivery, 1997, 12(1):51-60.

[11] Khan U. A. A multiprocessing platform for transient event detection [Thesis].Cambridge: Massachusetts Institute of Technology, 1995.

[12] Robert Cox S. B. L., Steven R. Shaw, Leslie K. Norford. Transient EventDetection for Nonintrusive Load Monitoring and Demand Side ManagementUsing Voltage Distortion [C] In: IEEE Applied Power ElectronicsConference and Exposition, 2006: 1751-1757. 